\title{
Análise dos Fatores Organizacionais Determinantes da Intenção de Rotatividade
}

\author{
Áurea de Fátima Oliveira ${ }^{1, *}$ \\ Orcid.org/0000-0001-5404-0540 \\ Sinésio Gomide Júnior ${ }^{1}$ \\ Orcid.org/0000-0003-0944-4380 \\ Bânia Vieira dos Santos Poli \\ Orcid.org/0000-0003-4364-5194 \\ Lígia Carolina Oliveira-Silva ${ }^{1}$ \\ Orcid.org/0000-0002-7487-9420 \\ ${ }^{l}$ Universidade Federal de Uberlândia, Uberlândia, $M G$, Brasil \\ ${ }^{2} I D E A$ - Instituto de Desenvolvimento Educacional Avançado, Escola Superior \\ de Administração, Marketing e Comunicação, Uberlândia, MG, Brasil
}

\section{Resumo}

Este estudo teve por objetivo investigar o papel mediador da confiança organizacional na relação entre percepção de suporte organizacional, percepção das políticas e práticas de gestão de pessoas e intenção de rotatividade. A amostra foi composta por 250 trabalhadores, predominando participantes do gênero feminino $(60,8 \%)$ com idade média de 28 anos e tempo médio de trabalho de 2,8 anos. A maioria estava empregada em organizações privadas $(89,25 \%)$ e possuía curso superior incompleto $(60,4 \%)$. Os dados foram analisados por meio de estatísticas descritivas e análise de caminhos - path analysis (Modelagem por Equações Estruturais), de forma a aferir as relações de mediação e efeitos indiretos entre as variáveis, analisando quais representam preditores diretos ou indiretos da intenção de rotatividade. Os resultados indicaram que o modelo proposto foi parcialmente corroborado, uma vez que as relações de mediação foram apenas parciais e os efeitos indiretos não foram significativos. Sugere-se a realização de estudos com outras amostras assim como a inclusão de outras variáveis nos modelos de investigação.

Palavras-chave: Intenção de rotatividade, percepção de suporte organizacional, políticas de gestão de pessoas, confiança organizacional.

* Endereço para correspondência: Rua Prof. Ciro de Castro Almeida, no 1905, bloco D - apto 22, Bairro Custódio Pereira, Uberlândia, MG, Brasil 38405-250.Fone: (34)99947-1205.E-mail: aurea.oliveira@ufu.br e ligiacarol@ ufu.br

Agradecimento à Fundação de Amparo à Pesquisa do Estado de Minas Gerais (FAPEMIG) pelo apoio financeiro na realização da pesquisa, a bolsista Ethyene Andrade Costa e a Fundação CDL - Câmara de Dirigentes Lojistas de Uberlândia. 


\title{
Analysis of Organizational Factors that Determine Turnover Intention
}

\begin{abstract}
The present study sought to examine the mediating role of organizational trust in the relationship between perceived organizational support, perception of personnel management policies and practices, and turnover intentions. The sample consisted of 250 employees, predominantly women (60.8\%), with a mean age of 28 years and an average length of employment of 2.8 years. Most of them was employed in private companies $(89.25 \%)$ and presented incomplete higher education $(60.4 \%)$. The data was analyzed through descriptive statistics and path analysis (Structural Equations Modeling) so as to appraise the mediation relationships and indirect effects between the variables, analyzing which of them represent direct or indirect predictors of turnover intentions. The results indicated that the proposed model was only partially corroborated, given that the mediation relationships were partial and the indirect effects were not significative. We recommend further studies involving both other samples and the inclusion of other variables in the research models.
\end{abstract}

Keywords: Turnover intention, perceived organizational support, personnel management policies, organizational trust.

\section{Análises de los Fatores Organizacionales Determinantes de la Intención de Rotatividad}

\section{Resumen}

Este estudio tuvo por objetivo investigar el papel mediador de la confianza organizacional en la relación entre percepción de soporte organizacional, percepción de las políticas y prácticas de gestión de personas e intención de rotatividad. La muestra fue compuesta por 250 trabajadores, predominando participantes del género femenino $(60,8 \%)$ con edad promedio de 28 años y tiempo medio de trabajo promedio de 2,8 años. La mayoría estava empleada en organizaciones privadas $(89,25 \%)$ y poseía curso superior incompleto $(60,4 \%)$. Los datos fueron analizados por medio de estadísticas descriptivas y análisis de caminos - path analysis (Modelación por Ecuaciones Estructurales), de forma a medir las relaciones de mediación y efectos indirectos entre las variables, analizando cuáles representan predictores directos o indirectos de la intención de rotatividad. Los resultados indicaron que el modelo propuesto fue parcialmente corroborado, ya que las relaciones de mediación sólo eran parciales y los efectos indirectos no fueron significativos. Se sugiere la realización de estudios con otras muestras así como la inclusión de otras variables en los modelos de investigación.

Palabras claves: Intención de rotatividad, percepción de soporte organizacional, políticas de gestión de personas, confianza organizacional.

O desligamento voluntário do trabalhador da empresa é um comportamento individual que se insere nos domínios do fenômeno de rotatividade de pessoal, podendo provocar repercussões como custos tangíveis (recrutamento, seleção, benefícios, treinamento, integração, desligamento) ou intangíveis (perda de know-how, quebra no fluxo de trabalho, quebra de vínculos com fornecedores e clientes, dentre outros). Também chamado de turnover, deve ser entendido como o número de funcionários desligados - por iniciativa própria ou da empresa - em determinado período (mensal ou anual) em comparação com o quadro de funcionários efetivos (Marras, 2000). Entretanto, o turnover que preocupa é aquele que ocorre a partir da decisão pessoal do trabalhador 
de deixar a organização em que trabalha, por ter efeitos adversos sobre a efetividade organizacional (Perez, 2008) e por dificultar o alcance das metas de produtividade das organizações (Flint, Haley, \& McNally, 2013; Kim, 2014; Rubel, Kee, Quah, \& Rimi, 2017). Adicionalmente, o desligamento voluntário pode ser considerado um obstáculo à manutenção de talentos, visando à busca da criação de valor para clientes, investidores, comunidade, fornecedores e acionistas (Flint et al., 2013). Logo, observa-se que os custos do turnover não são apenas financeiros, mas também psicológicos à medida que geram a desmoralização dos funcionários que permanecem e questionam se devem continuar na organização. Esses custos têm sido negligenciados por alguns gestores, porém o ambiente competitivo das organizações tem forçado o reconhecimento da necessidade de conhecer as causas do problema como condição prévia de seu controle (Flint et al., 2013). Lima, Céo, e Blatt (2016) consideram a insatisfação no trabalho causa de turnover, principalmente entre os jovens e aqueles insatisfeitos com o salário. Portanto, quando há melhores oportunidades no mercado de trabalho a tendência que prevalece é a intenção de buscar outras alternativas de trabalho. Diógenes, Paschoal, Neiva e Meneses (2016) identificaram que quanto menor a percepção de suporte organizacional, maior é a intenção de rotatividade no serviço público. Anteriormente Siqueira e Gomide, em 2014, já haviam assinalado a importância desse suporte em relação à intenção do empregado em permanecer, ou não, na organização, seja ela pública ou privada.

Diferente do turnover, a Intenção de Rotatividade (IR) capta a percepção e a avaliação do indivíduo a respeito de alternativas de trabalho (Perez, 2008). Trata-se de um fenômeno de natureza psicológica que, na concepção de Mowday, Porter e Steers (1982), se referem à probabilidade estimada (subjetiva) própria dos indivíduos de que eles estão permanentemente deixando a organização em algum ponto do futuro próximo. A intenção de rotatividade tornou-se interesse de pesquisa no campo do comportamento organizacional por ser um indicador eficaz para avaliar o pensamento, o planejamento e a vontade de trabalhadores deixararem a organização de trabalho. Entretanto, neste estudo não será estudada de forma direta a rotatividade de pessoal em si, pois o interesse é investigar quais as razões que levam as pessoas a terem a intenção de deixar as organizações em que trabalham (Mintzberg \& Lampel, 1999). Em geral, as empresas raramente disponibilizam dados brutos relativos à movimentação de pessoal; inclusive muitas delas sequer dispõem desse controle. Ademais, é possível considerar que a intenção comportamental é um forte preditor do comportamento (Fishbein \& Ajzen, 1975, citados por Flint et al., 2013). O modelo proposto por Mobbley (1977, citado por Khan, 2014) esclarece o mecanismo psicológico do fenômeno que, como processo, segue algumas etapas: avaliação do trabalho, vivência de insatisfação, pensamento de sair, avaliação da expectativa da utilidade da busca e custo da saída, intenção de buscar alternativas, busca de alternativas, avaliação dessas alternativas, comparação das alternativas com o trabalho atual, intenção de sair/ficar, e sair ou ficar. Considerando este processo a definição proposta por Tett e Meyer (1993) refere-se à disposição consciente e deliberada de deixar a organização.

A produção científica sobre intenção de rotatividade tem crescido nos últimos anos (Agapito, 2012), de forma que 112 artigos internacionais foram publicados no período de 2007 a 2012, especialmente em revistas da área de administração, sendo 37 classificados no Qualis Capes. Segundo Agapito (2012) a produção nacional mostrou-se menor que a internacional (11 artigos), dos quais somente 7 são classificados no Qualis, prevalece ainda as revistas da área da administração. Esse panorama geral reafirma a pertinência do tema já abordado por autores anteriores a esse período, juntamente com a ampliação das variáveis investigadas.

A busca dos fatores geradores da rotatividade já se fazia notar no estudo de Brannick (1999), no qual a rotatividade se manifesta em função da relação deficiente entre o funcionário e a cultura organizacional, do treinamento inadequado, da falta de incentivos e das políticas organizacio- 
nais. Siqueira e Gomide, em 2014, acrescentam que oportunidades de progresso profissional oferecida pela empresa, tipo de supervisão, a política disciplinar, as condições ambientais de trabalho e a motivação do pessoal também podem gerar rotatividade.

A investigação das variáveis antecedentes da intenção de rotatividade, portanto, poderia oferecer às empresas possibilidades de delinear ações no intuito de reter talentos importantes para o alcance da sua efetividade. Os antecedentes mais investigados nas literaturas nacionais e internacionais têm sido o comprometimento organizacional afetivo em relação negativa com intenção de rotatividade (Blomme, Van Rheede, \& Tromp, 2010; Lima et al., 2016; Siqueira \& Gomide, 2014; Yin-Fah, Foon, Chee-Leong, \& Osman, 2010); clima ético (Rubel et al., 2017); valores éticos (Valentine, Godkin, Fleischman, \& Kidwell, 2010); cinismo organizacional (Khan, 2014), práticas de recursos humanos (Juhdi, Pa'wan, \& Hansaram, 2013; Knap, Smith, \& Sprinkle, 2017) e, por fim, justiça organizacional e confiança (Farooq \& Farooq, 2014).

Outro preditor importante apontado pela literatura é a percepção de suporte organizacional (Diógenes et al., 2016; Siqueira \& Gomide, 2014), cujas consequências são menos faltas ao trabalho, maior desempenho, maior satisfação no trabalho e menor intenção de sair da empresa. Além da diminuição da intenção de sair da organização, a Percepção de Suporte Organizacional (PSO) é preditora de maior comprometimento afetivo, comportamento de cidadania organizacional, melhor desempenho, menor necessidade de supervisão, criatividade e inovação e confiança organizacional (Batista, \& Oliveira, 2012; Eisenberg, Huntington, Hutchison, \& Sowa, 1986; Manzoor, \& Naeem, 2011; Siqueira \& Gomide, 2014). Contudo, há necessidade de ampliar as investigações resgatando outras variáveis que possam afetar a intenção de rotatividade.

Neste sentido, as Políticas e Práticas de Gestão de Pessoas (PPGP) têm ganhado espaço nas investigações nacionais e internacionais, conforme ilustrado pelos estudos de Horta,
Demo e Roure (2012) e Shuck, Twyford, Reio e Shuch (2014), centrados nas práticas de gestão de pessoas, respectivamente, com comprometimento organizacional e intenção de rotatividade, e confiança organizacional e bem-estar no trabalho. Para Dall'Inha (2006), a ocorrência da rotatividade pode ser a ponta do iceberg de distorções nas políticas de recursos humanos (RH). A análise da rotatividade pode, por exemplo, influenciar a reformulação das políticas de $\mathrm{RH}$ das organizações.

Por fim, uma variável ainda pouco explorada em sua relação com a intenção de rotatividade é a confiança organizacional, que está associada a resultados como comprometimento organizacional, comportamento de cidadania organizacional, maior cooperação e menor necessidade de controle (Kramer, 1999). Farooq e Farooq (2014) demonstraram que os empregados são sensíveis as questões relativas à justiça no contexto de trabalho e verificaram que confiança organizacional medeia a relação entre percepção de justiça e intenção de turnover. Assim as percepções de justiça distributiva e de procedimento aumentam a confiança do empregado na organização diminuindo a intenção de rotatividade. Outro aspecto que afeta essa intenção é percepção de suporte organizacional (Diógenes et al., 2016). Assim, há indicativos que a relação entre PSO, e percepção de práticas e políticas de gestão de pessoas e intenção de rotatividade é mediada pela confiança na organização, sendo esta uma das hipóteses a ser testada neste estudo.

Em síntese, a literatura indica que a PSO e a PPGP e confiança organizacional podem predizer a intenção de rotatividade, sendo possível que a confiança, por sua vez, desempenhe papel mediador na relação. Embora não tenham sido encontrados estudos que tratassem da confiança organizacional como antecedente da intenção de rotatividade, foi encontrado, por outro lado, suporte na literatura para sua inclusão como mediadora em um modelo de investigação de variáveis análogas àquelas aqui utilizadas. Logo, o presente estudo apresentou como principal objetivo o teste do seguinte modelo (vide Figura 1). 


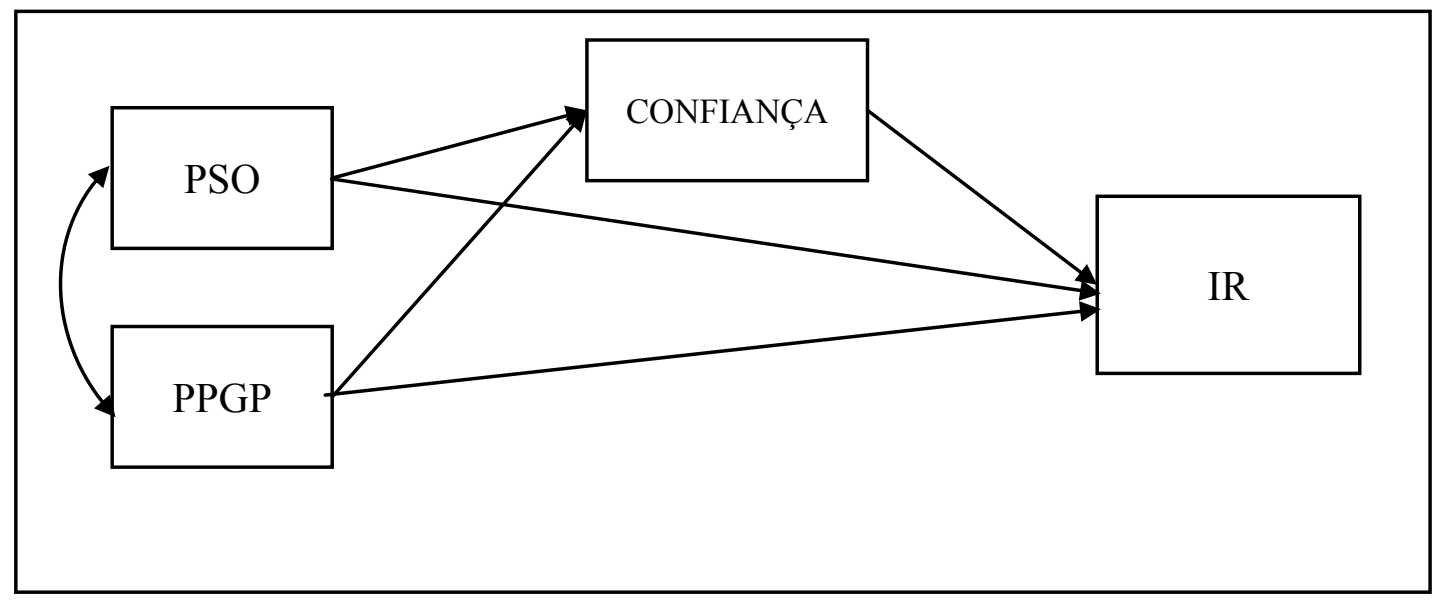

Figura 1. Modelo teórico da pesquisa.

\section{Método}

\section{Participantes}

A amostra de conveniência foi composta por 250 trabalhadores vinculados a organizações formais em uma cidade do Triângulo Mineiro (MG), dos quais $60,8 \%$ correspondem ao gênero feminino. Predominaram participantes com ensino superior incompleto $(60,4 \%)$ e vinculados a organizações privadas $(89,2 \%)$. A média da foi de 28 anos $(\mathrm{DP}=10,21)$ e o tempo médio de trabalho é de 2,89 anos ( $\mathrm{DP}=5,75$ anos). O número de participantes foi obtido através do cálculo do poder do teste para indicação do tamanho da amostra, considerando o efeito médio $\left(\mathrm{f}^{2}=0,15\right.$; $\mathrm{p}<0,05)$, que indicou, no mínimo, 172 casos como necessários.

\section{Instrumentos}

Escala de Intenção de Rotatividade (Siqueira, Gomide, Oliveira, \& Polizzi, 2014) - o instrumento é composto por três itens, com índice de precisão (Alpha) de 0,89 - "Você planeja sair da empresa onde trabalha". Os participantes utilizam uma escala de cinco pontos que revela a frequência de cada afirmação, variando de 1 (nunca) a 5 (sempre) para se manifestarem. Escala Percepção de Políticas de Gestão de Pessoas (Demo, Neiva, Nunes, \& Rozzet, 2014) - as políticas de gestão de pessoas referem-se a recrutamento e seleção (seis itens - $\alpha=0,84$ ) - "Os processos seletivos da organização onde eu tra- balho são disputados, atraindo pessoas competentes"; envolvimento ( 12 itens, $\alpha=0,93$ ) - "A organização onde eu trabalho estimula a minha participação nas tomadas de decisão e resolução de problemas"; treinamento, desenvolvimento e educação (seis itens, $\alpha=0,88$ ) - "Eu consigo aplicar no meu trabalho os conhecimentos e comportamentos aprendidos nos treinamentos/ eventos de que participo"; condições de trabalho (seis itens, $\alpha=0,84$ ) - "Na organização onde eu trabalho, existem ações e programas de prevenção de acidentes e enfrentamento de incidentes"; avaliação de desempenho e competências (cinco itens, $\alpha=0,86$ ) - "A organização onde eu trabalho realiza avaliações de desempenho e competências periodicamente" e recompensas (cinco itens, $\alpha=0,81)$ - "Na organização onde eu trabalho, minha remuneração é influenciada pelos meus resultados". Os participantes usam escala de resposta tipo Likert de cinco pontos para indicar o quanto concordam ou discordam de cada afirmação, variando entre discordo totalmente da afirmativa (1) e concordo totalmente com a afirmativa (5).

Inventário de Confiança Organizacional (Ianaguivara, 2011) - é composto por três fatores: componentes éticos (23 itens, $\alpha=0,96)$ - "Nesta organização, é possível perceber a boa vontade em alcançar objetivos favoráveis a todos"; competência organizacional (14 itens, $\alpha=0,92$ ) - "Esta organização adota novas tecnologias mantendo-se competitiva" e oportunismo, com 
(cinco itens, $\alpha=0,84)$ - "Esta organização se aproveita dos erros de outros para levar vantagem". Os participantes usam a escala de resposta tipo Likert para indicar sua concordância ou discordância quanto ao conteúdo de cada afirmação variando de 1 (discordo totalmente) a 5 (concordo totalmente).

Escala de Percepção de Suporte Organizacional (Siqueira \& Gomide, 2008) - É constituída por seis itens que expressam crenças positivas sobre apoio e valorização do empregado pela organização $(\alpha=0,86)$ - "É possível obter ajuda desta empresa quanto tenho um problema”. Os participantes utilizam uma escala de resposta Likert de sete pontos variando de discordo totalmente (1) a concordo totalmente (7).

\section{Procedimentos de Coleta de Dados, Éticos e Análise de Dados}

Para o alcance dos objetivos foram convidados trabalhadores que atendiam às seguintes condições: idade superior a 18 anos, estarem empregados em uma organização formal há mais de seis meses e possuir ensino fundamental completo, o que possibilitaria a leitura e a compreensão dos itens dos instrumentos. Os voluntários responderam aos questionários, após o preenchimento do Termo de Consentimento Livre e Esclarecido em ambientes abertos ou por intermédio da área de recursos das empresas que distribuiu e recolheu os questionários. $\mathrm{O}$ projeto no qual está inserido este estudo foi aprovado no Comitê de Ética e Pesquisa, conforme Resolução no 466/2012, do Conselho Nacional de Saúde, com Parecer no 197.721.

Os dados foram analisados com o suporte dos programas SPSS - Statistical Package of Social Science e AMOS. A amostra foi analisada por meio de estatísticas descritivas (média, frequência, desvio padrão) enquanto as correlações entre as variáveis foram verificadas através da correlação de Pearson. Para o teste do modelo, isto é, verificação das relações de predição e de mediação, foi executada a path analysis por meio da modelagem de equações estruturais, além do procedimento de bootstrapping para verificação dos efeitos indiretos. O método de estimação uti- lizado foi o de máxima verossimilhança ou maximum likelihood.

\section{Resultados}

Na preparação do arquivo de dados observou-se que não havia erros de digitação e os dados omissos foram inferiores a $5 \%$ da amostra (2,4\%). Em consonância com Pasquali (2015) e Tabachnick e Fidell (2001), os dados omissos foram substituídos pela média da amostra. Os casos univariados de outliers, por serem moderados, foram mantidos na amostra, mas os multivariados, em número de 19, identificados por meio da distância de Mahalanobis (Pasquali, 2015; Tabachnick \& Fidell, 2001) e, com base no $\chi^{2}$ calculado $\left(\chi^{2}=137,216 ; \mathrm{gl}=90 ; \mathrm{p}<0,001\right)$, foram eliminados da amostra, que passou a ter 231 participantes.

A normalidade dos dados foi verificada por meio dos índices de assimetria e curtose, tendo em vista os parâmetros definidos por Miles e Shevlin (2001), que consideram aceitáveis os índices entre 1 e 2 . Verificou-se a multicolinearidade por meio da matriz de correlação, dos valores da tolerância e do fator de inflação da variância (FIV) vistos nos resultados da análise de regressão. De acordo com a matriz de correlação (Tabela 1), não foi observada na maioria das variáveis a existência de multicolinearidade, considerando que os coeficientes de correlação encontrados foram inferiores a 0,80 , como indicado por Field (2009), exceto na correlação entre políticas de envolvimento e confiança na ética organizacional $(r=0,84)$. Uma vez que os valores da tolerância menores que 0,10 e valores de FIV maiores que 10 são indicativos de multicolinearidade, neste estudo, os resultados não indicaram problemas graves de multicolinearidade entre as variáveis estudadas. Entretanto, a partir da existência de correlações moderadas e fortes na matriz de correlação, foi realizada a centralização das variáveis preditoras, de forma a diminuir eventuais efeitos de colinearidade nos parâmetros da análise de caminhos. 


\section{Análise Descritiva dos Dados}

A descrição estatística das variáveis estudadas foi realizada por meio das médias fatoriais, conforme as instruções dos respectivos autores das escalas. A Tabela 1 apresenta os resultados. As correlações entre intenção de rotatividade e demais variáveis são negativas e significativas, exceto com oportunismo cuja direção da relação é positiva. Percepção de suporte organizacional (PSO), por sua vez, mantém correlação negativa somente com oportunismo $(r=-0,25 ; p<0,01)$.
Há uma associação entre esta percepção e confiança na ética e competências organizacionais assim como as políticas de gestão de pessoas. Os maiores índices foram observados entre PSO e confiança na ética $(r=0,72 ; p<0,01)$ e com políticas de envolvimento $(r=0,73 ; p<0,01)$. Confiança na ética mantém correlação elevada com políticas de envolvimento $(r=0,84 ; p<0,01)$, sendo as demais de menor magnitude. Destaca-se que oportunismo apresenta uma única correlação negativa significativa com políticas de envolvimento.

Tabela 1

Estatística Descritiva e Confiabilidade das Escalas para a Amostra do Estudo

\begin{tabular}{|c|c|c|c|c|c|c|c|c|c|c|c|c|c|}
\hline & M & DP & 1 & 2 & 3 & 4 & 5 & 6 & 7 & 8 & 9 & 10 & 11 \\
\hline 1. IR & 2,55 & 1,39 & $(0,92)$ & & & & & & & & & & \\
\hline 2. PSO & 4,84 & 1,37 & $-0,45^{* *}$ & $(0,87)$ & & & & & & & & & \\
\hline 3. C.ÉTICOS & 3,48 & 0,80 & $-0,51^{* *}$ & $0,72^{* *}$ & $(0,96)$ & & & & & & & & \\
\hline 4. C.ORG & 4,05 & 0,66 & $-0,38^{* *}$ & $0,39^{* *}$ & $0,64^{* *}$ & $(0,93)$ & & & & & & & \\
\hline 5. OPORT. & 2,31 & 0,83 & $0,29^{* *}$ & $-0,25^{* *}$ & $-0,36^{* *}$ & $-0,29^{* *}$ & $(0,84)$ & & & & & & \\
\hline 6. $\mathrm{R} \& \mathrm{~S}$ & 3,66 & 1,03 & $-0,37^{* *}$ & $0,45^{* *}$ & $0,52^{* *}$ & $0,41^{* *}$ & $-0,09$ & $(0,86)$ & & & & & \\
\hline 7. ENVOLV. & 3,61 & 0,84 & $-0,51^{* *}$ & $0,73^{* *}$ & $0,84^{* *}$ & $0,53^{* *}$ & $-0,29^{* *}$ & $0,58^{* *}$ & $(0,91)$ & & & & \\
\hline 8. TD\&E & 3,26 & 1,08 & $-0,47^{* *}$ & $0,59^{* *}$ & $0,65^{* *}$ & $0,45^{* *}$ & $-0,07 \mathrm{~ns}$ & $0,67^{* *}$ & $0,74^{* *}$ & $(0,90)$ & & & \\
\hline 9. COND.TRAB. & 3,55 & 0,87 & $-0,31^{* *}$ & $0,37^{* *}$ & $0,53^{* *}$ & $0,52^{* *}$ & $0,00 \mathrm{~ns}$ & $0,61^{* *}$ & $0,51^{* *}$ & $0,62^{* *}$ & $(0,75)$ & & \\
\hline 10. AD\&COMPT & 3,02 & 1,24 & $-0,37^{* *}$ & $0,49^{* *}$ & $0,61^{* *}$ & $0,36^{* *}$ & $0,01 \mathrm{~ns}$ & $0,60^{* *}$ & $0,63^{* *}$ & $0,75^{* *}$ & $0,55^{* *}$ & $(0,93)$ & \\
\hline 11. RECOMP. & 3,26 & 1,09 & $-0,45^{* *}$ & $0,53^{* *}$ & $0,65^{* *}$ & $0,47^{* *}$ & $-0,12 \mathrm{~ns}$ & $0,53^{* *}$ & $0,67^{* *}$ & $0,63^{* *}$ & $0,48^{* *}$ & $0,64^{* *}$ & $(0,83)$ \\
\hline
\end{tabular}

Nota. $\mathrm{n}=231 ; \mathrm{IR}=$ intenção de rotatividade; $\mathrm{PSO}=$ Percepção de Suporte Organizacional; C.ÉTICOS = Componentes éticos; C.ORG = Competência Organizacional; OPORT. $=$ Oportunismo; $R \& S=$ Políticas de gestão de pessoas de recrutamento e seleção; ENVOLV. = Políticas de gestão de pessoas de envolvimento; TD\&E = Políticas de gestão de pessoas de treinamento, desenvolvimento e educação; COND.TRAB. = Políticas de gestão de pessoas de condições de trabalho; AD\&COMPT = Políticas de gestão de pessoas de avaliação de desempenho e competências; RECOMP = Políticas de gestão de pessoas de recompensas. Os números na diagonal são os valores do Alpha de Cronbach.

$* * \mathrm{p}<0,001, \mathrm{~ns}=$ não significativo

\section{Teste do Modelo}

Para o teste da hipótese de mediação e avaliação geral do ajuste, o modelo proposto na Figura 1 foi analisado através da modelagem por equações estruturais - path analysis. Considerando a estrutura fatorial dos instrumentos utilizados, o modelo especificado fez uso de variáveis latentes, no caso de PPGP e confiança, por serem multifatoriais, e variáveis observadas para IR e PSO, por serem unifatoriais, conforme Figura 2.

O modelo inicial especificado apresentou índices de ajuste aceitáveis $(\mathrm{CMIN} / \mathrm{DF}=5,87$, $\mathrm{CFI}=0,88, \mathrm{GFI}=0,84, \mathrm{TLI}=0,83, \mathrm{NFI}=0,86$ atendendo ao mínimo estabelecido por Marôco (2014). Porém, os resíduos encontrados foram relativamente altos $(\mathrm{RMSEA}=0,16$; SRMR $=0,074)$. A partir dos índices de modificação 


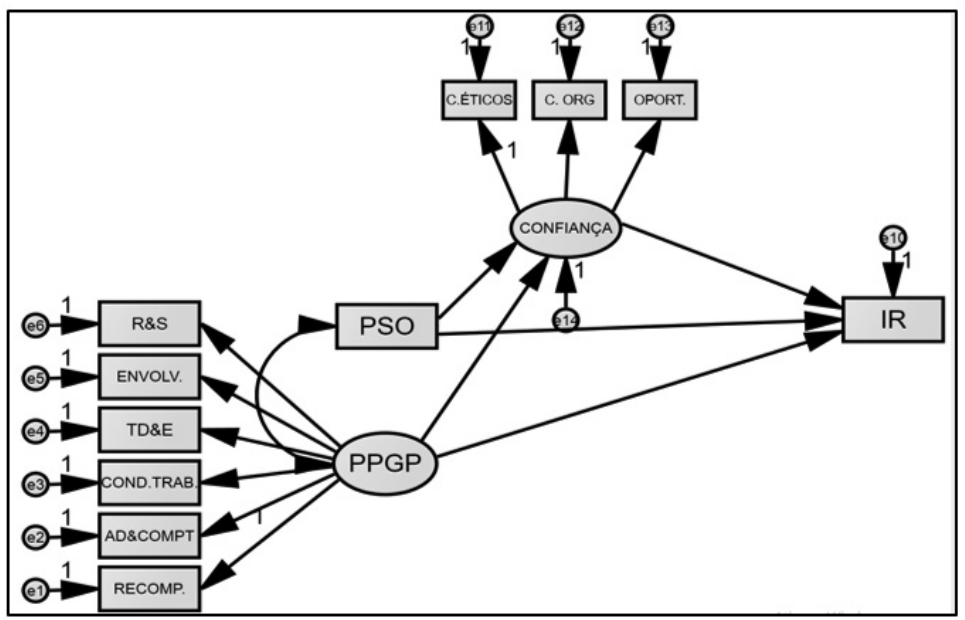

Figura 2. Modelo especificado na path analysis.

sugeridos pelo AMOS, foram traçadas correlações entre os erros os seguintes fatores: $R \& S$ - COND.TRAB., ENVOLV. - AD\&COMPT., TD\&E - AD \& COMPT. Segundo Kline (2010), algumas correlações entre erros podem ser aceitáveis, uma vez que melhorem o ajuste do modelo. O modelo ajustado apresentou melhora nos índices de ajuste $(\mathrm{CMIN} / \mathrm{DF}=4,75, \mathrm{CFI}=0,91$, $\mathrm{GFI}=0,88, \mathrm{TLI}=0,87, \mathrm{NFI}=0,90)$, e embora os resíduos tenham diminuído, os valores alcançados ainda estariam acima do desejável (RM$\mathrm{SEA}=0,12 ; \mathrm{SRMR}=0,071$ ).

Após a análise de ajuste do modelo, foram observados os coeficientes que permitem o teste de hipótese do modelo de mediação. Para tanto, foram observados os efeitos diretos das variáveis independentes sem a mediadora, na presença da mediadora e os efeitos indiretos, conforme Tabela 2.

Tabela 2

Resultado do Teste de Hipótese para a Variável Dependente IR

\begin{tabular}{cccc}
\hline Relações testadas & $\begin{array}{c}\text { Efeitos diretos } \\
\text { sem a mediadora }\end{array}$ & $\begin{array}{c}\text { Efeitos diretos } \\
\text { com a mediadora }\end{array}$ & $\begin{array}{c}\text { Efeitos indiretos } \\
\text { com Bootstraping }\end{array}$ \\
\hline PSO à CONF à IR & $-0,233^{*}$ & $-0,193^{*}$ & NS \\
PPGP à CONF à IR & $-0,337 * *$ & $-0,276^{*}$ & NS \\
\hline
\end{tabular}

$* \mathrm{p}<0,05 ; * * \mathrm{p}<0,001 ; \mathrm{NS}=$ não significativo.

No caso dos efeitos diretos na presença da mediadora, é possível identificar uma mediação parcial, uma vez que o coeficiente apresenta decréscimo estatisticamente significativo diante da inclusão da confiança na equação. Entretanto, os resultados obtidos para os efeitos indiretos não foram significativos, o que reforça a conclusão da existência de mediação parcial. Logo, é possível afirmar que existe efeito de mediação, embora tal efeito não anule os efeitos das relações diretas, uma vez que os efeitos indiretos não são significativos. A partir da Tabela 3, é possível analisar os coeficientes de todas as relações analisadas, com destaque para a relação não significativa entre a variável mediadora confiança e a variável dependente, intenção de rotatividade. Tal resultado pode representar um dos motivos pelos quais a hipótese de mediação foi apenas parcialmente apoiada, pois segundo Baron e Kenny (1986), para que haja mediação, é necessário que a variável mediadora anteceda a variável critério, o que não é demonstrado pelos re- 
sultados. PSO e PPGP, por sua vez, apresentam relações de predição significativas tanto com a confiança organizacional quanto com a intenção de rotatividade, entretanto, PSO e PPGP predizem positivamente a confiança, enquanto predizem negativamente a intenção de rotatividade.

Tabela 3

Coeficientes das Relações Obtidas na Path Analysis

\begin{tabular}{lcc}
\hline \multicolumn{1}{c}{ Relação de variáveis } & Coeficiente padronizado & p-value \\
\hline PSO à CONFIANÇA & 0,164 & $*$ \\
PPGP à CONFIANÇA & 0,709 & $* *$ \\
PPGP à RECOMP. & 0,738 & $* *$ \\
PPGP à AD\&COMPT. & 0,745 & $* *$ \\
PPGP à COND.TRAB. & 0,603 & $* *$ \\
PPGP à TD\&E & 0,796 & $* *$ \\
PPGP à ENVOLV. & 0,932 & $* *$ \\
PPGP à R\&S & 0,652 & $* *$ \\
PSO à IR & $-0,201$ & $*$ \\
PPGP à IR & $-0,251$ & $*$ \\
CONFIANÇA à C.ETICOS & 0,515 & $* *$ \\
CONFIANÇA à C.ORG & 0,621 & $* *$ \\
CONFIANÇA à OPORT. & $-0,360$ & $* *$ \\
CONFIANÇA à IR & $-0,122$ & 0,206 \\
\hline
\end{tabular}

${ }^{*} \mathrm{p}<0,05 ; * * \mathrm{p}<0,001$

\section{Discussão}

Os resultados indicaram que as direções das correlações se mostraram conforme as indicações da literatura. Intenção de rotatividade (variavel - critério) mantém correlações negativas com PSO (Siqueira \& Gomide, 2014), percepção de políticas e práticas de gestão e confiança na ética e competência organizacionais. A intenção de deixar a organização se dá a medida que o empregado não percebe suporte desta ou PPGP que sejam efetivamente praticadas. Benjamin (2012) identificou relação negativa significativa entre clima de desenvolvimento de recursos humanos e intenção de turnover.

A síntese da literatura apresentada por Siqueira e Gomide (2014) apresenta como preditores da intenção de rotatividade, a percepção de suporte organizacional (PSO), comprometimento organizacional (afetivo, calculativo e nor- mativo), satisfação no trabalho e percepções de justiça de procedimentos e distributiva. Manzoor e Naeem (2011), por sua vez, identificaram que se o empregado percebe suporte da organização, eles relatam maiores níveis de comprometimento e menor intenção de sair da organização.

Em relação a confiança organizacional, a literatura sugere que, se há confiança, o empregado se percebe menos vulnerável às ações da outra parte (Kramer, 1999). Por se tratar de uma troca social, a confiança não se utiliza de controles formais, porém, ainda assim permite uma certa tranquilidade nas relações sociais (Kramer, 1999). Há o pressuposto que as promessas serão cumpridas, não haverá deslealdade e qualquer forma de prejuízo para aquele que deposita sua confiança em outrem. Ao se observar os coeficientes de regressão, nota-se que as variaveis PSO e PPGP se posicionaram como preditoras da confiança organizacional. Resultados simila- 
res foram encontrados anterioresmente na literatura, uma vez que estudos de Horta et al. (2012) e Shuck et al. (2014) relacionaram as práticas de gestão de pessoas com comprometimento organizacional, engajamento no trabalho, intenção de rotatividade, confiança na organização e bem-estar no trabalho.

Ainda considerando os coeficientes, observa-se que PSO e PPGP também aparecem como antecedentes significativos da intenção de rotatividade, porém com coeficientes negativos, o que indica que quanto maior a percepção de suporte, aliada à percepção mais positiva das políticas $\mathrm{e}$ práticas de gestão de pessoas, menor será a intenção de rotatividade; percepções negativas de suporte e de gestão, por outro lado, podem estimular o desejo de desligamento da organização. Embora o modelo teórico proposto apresente índices de ajuste aceitáveis, a hipótese de mediação foi apenas parcialmente apoiada. A confiança organizacional, por sua vez, não prediz significativamente a intenção de rotatividade, o que pode ajudar a compreender porque a mediação não é completa, assim como a não-significância dos efeitos indiretos. Portanto, embora a percepção de suporte e das políticas e práticas de gestão de pessoas afetem a confiança do empregado na organização, não é possível afirmar que a ausência desta seja o principal motivo pelo qual o empregado deseja deixar a organização, ou que chegue a anular o impacto do suporte e da gestão de pessoas.

$\mathrm{Na}$ concepção de Gouldner (1960), a norma de reciprocidade ('as pessoas devem ajudar aquelas que as ajudaram; as pessoas não devem prejudicar seus benfeitores') oferece aos indivíduos uma base realista para a confiança. A norma de reciprocidade, ao estimular os atores a desempenharem seus papéis conforme a expectativa existente, também corrobora o estabelecimento da confiança. Uma vez ferida esta norma a base da relação de trabalho se torna frágil e não compensadora. Do outro lado, a falta de investimento da organização no crescimento/ aprimoramento profissional de seus colaboradores pode ser vista como indicativo da pouca valorização desses. Assim, os resultados parecem indicar que se o empregado não confia nas ações organiza- cionais, mas também não percebe suporte e boas práticas de gestão, a melhor alternativa seria procurar outra organização. Estas crenças, quando encontram condições propícias no mercado de trabalho, levariam ao desligamento voluntário do empregado. É claro que a possibilidade do encadeamento descrito de crenças e cognições poderia explicar os resultados finais deste estudo. Ressalta-se que o papel da confiança, como variável mediadora, moderadora ou antecedente ainda permanece merecendo melhores esclarecimentos por meio de novas investigações.

De qualquer modo, é preciso investigar a proposição do modelo testado em outras amostras e regiões do país, ampliando sua capacidade de generalização. A solidez do conhecimento depende do teste empírico de modelos em outras realidades. Contudo, de imediato, os gestores têm indicações sobre aspectos importantes que podem auxiliar no gerenciamento de pessoas, especialmente, visando à retenção de talentos caros às organizações.

\section{Referências}

Agapito, P. R. (2012). Bem-estar no trabalho e percepção de sucesso na carreira como antecedentes de intenção de rotatividade (Dissertação de mestrado não publicada, Universidade Metodista de São Paulo, São Bernardo do Campo, SP, Brasil).

Baron, J. B., \& Kenny, D. A. (1986). The moderatormediator variable distinction in social psychology research: Conceptual, strategic, an statistical considerations. Journal of Personality and Social Psychology, 51(6), 1173-1182. doi: http:// dx.doi.org/10.1037/0022-3514.51.6.1173

Batista, R. L., \& Oliveira, A. F. (2012). Antecedentes da confiança do empregado na organização. Estudos de Psicologia (Natal), 17(2), 247-254.

Benjamin, A. (2012). Human resource development climate as a predictor of citizenship behaviour and voluntary turnover intentions in the banking sector. International Business Research, 5(1), 110-119. doi: http://dx.doi.org/10.5539/ibr. v5n $1 \mathrm{p} 110$

Blomme, R. J., Van Rheede, A., \& Tromp, D. M. (2010). The use of the psychological contract to explain turnover intentions in the hospitality 
industry: A research study on the impact of gender on the turnover intentions of highly educated employees. International Journal of Human Resource Management, 21(1), 144-162. doi: $10.1080 / 09585190903466954$

Brannick, J. (1999). Decreasing the staggering costs of turnover in your organization. On line. Available, 1999. Retrieved from http://www.proquest.com

Dall'Inha, G. R. (2006). A influência das práticas e das políticas de recursos humanos sobre o absenteísmo e a rotatividade: Um estudo de caso (Dissertação de mestrado não publicada, Universidade Federal de Santa Catarina, Florianópolis, SC, Brasil).

Demo, G., Neiva, E. R., Nunes, I., \& Rozzet, K. (2014). Políticas e práticas de recursos humanos. In M. M. M. Siqueira (Ed.), Novas medidas do comportamento organizacional: Ferramentas de diagnóstico e de gestão (pp. 240-255). Porto Alegre, RS: Artmed.

Diógenes, L. C., Paschoal, T., Neiva, E. R., \& Meneses, P. P. M. (2016). Intenção de rotatividade e percepção de suporte organizacional em um órgão público. Revista Serviço Público, 67(2), 147-172. doi: https://doi.org/10.21874/rsp. v67i2.655

Eisenberg, R., Huntington, R., Hutchison, S., \& Sowa, D. (1986). Perceived organizational support. Journal of Applied Psychology, 17(3), 500-507.

Farooq, M., \& Farooq, O. (2014). Organizational justice, employee turnover, and trust in the workplace: A study in South Asian telecommunication campanies. Global Business and Organizational Excellence, 33(3), 56-62. doi: $10.1002 /$ joe. 21539

Field, A. (2009). Descobrindo a estatística usando o SPSS. Porto Alegre, RS: Penso.

Flint, D., Haley, L. M., \& McNally, J. J. (2013). Individual and organizational determinants of turnover intent. Personnel Review, 42(5), 552-572. doi: 10.1108?PR-03-2012-0051

Gouldner, A. W. (1960). The norm of reciprocity: A preliminary statement. American Sociological Review, 25(2), 161-178.

Horta, P., Demo, G., \& Roure, P. (2012). Políticas de Gestão de Pessoas, Confiança e Bem-estar no trabalho: Estudo em uma multinacional. Re- vista de Administração Contemporânea, 16(4), 566-585. doi: http://dx.doi.org/10.1590/S141565552012000400005

Ianaguivara, C. M. G. A. (2011). Confiança da empregado na organização: Revalidação de instrumento de medida (Dissertação de mestrado não publicada, Universidade Federal de Uberlândia, MG, Brasil).

Juhdi, N., Pa'wan, F., \& Hansaram, R. M. K. (2013). HR practices and turnover intention: The mediating roles of organizational commitment and organizational engagement in a selected region in Malaysia. The International Journal of Human Resource Management, 24(15), 3002-3019. doi: 10.1080/09585192.2013.763841

Khan, M. A. (2014). Organizational cynicism and employee turnover intention: Evidence from banking sector in Pakistan. Pakistan Journal of Commerce and Social Sciences, 8(1), 30-41.

Kim, N. (2014). Employee turnover intention among newcomers in travel industry. International Journal of Tourism Research, 16(1), 56-64. doi: $10.1002 /$ jtr. 1898

Kline, R. B. (2010). Principles and Practice of Structural Equation Modeling. New York: Guilford Press.

Kramer, R. M. (1999). Trust and distrust in organizations: Emerging perspective, enduring questions. Annual Review of Psychology, 50, 569598. doi: 10.1146/annurev.psych.50.1.569

Lima, B. F., Céo, M., \& Blatt, N. (2016, 17-21 out.). Fatores influenciadores da rotatividade de servidores efetivos e temporários: Estudo de uma instituição pública. Anais SEMAD XVIII, 3(1), $1-15$.

Manzoor, M., \& Naeem, H. (2011). Relationship of organization socialization with organizational commitment and turnover intention: Moderating role of perceived organizational support, interdisciplinary. Journal of Contemporary Research in Business, 3(8), 515-529. doi: http://www. journal-archieves13.webs.com/515-529.pdf

Marôco, J. (2014). Análise de equações estruturais: Fundamentos teóricos, software \& aplicações. Pêro Pinheiro, Portugal: Cafilesa.

Marras, J. P. (2000). Administração de Recursos Humanos: Do operacional ao estratégico. São Paulo, SP: Futura. 
Miles, J., \& Schevlin, M. (2001). Applying regression \& correlation. A guide for students e researchers. London: Sage.

Mintzberg, H., \& Lampel, J. (1999). Reflecting on the Strategy Process. Sloam Management Review, 40(3), 21-30.

Mowday, R. T., Porter, L. W., \& Steers, R. M. (1982). Employee-organization linkages: The psychology of commitment, absenteeism, and turnover. New York: Academic Press.

Pasquali, L. (2015). Delineamento de pesquisa em ciência: Fundamentos estatísticos da pesquisa científica (Vol. 2). São Paulo, SP: Vetor.

Perez, M. (2008). Turnover intention (Doctoral dissertation, University of Zurich, Brüttisellen, Germany)

Rubel, M. R. B., Kee, D. M. H., Quah, C. H., \& Rimi, N. N. (2017). Ethical climate and employee turnover intention in the ready-made garment industry of Bangladesh. Global Business and Organizational Excellence, 36(2), 61-73. doi: $10.1002 /$ joe

Shuck, B., Twyford, D., Reio, T. G., Jr., \& Shuck, A. (2014). Human resource development practices and employee engagement: Examining the connection with employee turnover intentions. Human Resource Development Quartely, 25(2), 113-144. doi: 10.1002/hrdq.21190

Siqueira, M. M. M., \& Gomide, S., Jr. (2008). Suporte no trabalho. In M. M. M. Siqueira (Ed.), Medidas do comportamento organizacional: Ferramentas de diagnóstico e de gestão (pp. 283-294). Porto Alegre, RS: Artmed.

Siqueira, M. M. M., \& Gomide, S., Jr. (2014). Vínculos do Indivíduo com o Trabalho e com a Organização. In J. E. Zanelli, J. E. Borges-Andrade, \& A. V. B. Bastos (Eds.), Psicologia, organizações e trabalho no Brasil (pp. 316-350). Porto Alegre, RS: Artmed.
Siqueira, M. M. M., Gomide, S., Jr., Oliveira, A. F. \& Polizzi, A., Filho. (2014). Intenção de rotatividade. In M. M. M. Siqueira (Ed.), Medidas do comportamento organizacional: Ferramentas de diagnóstico e de gestão (pp. 209-216). Porto Alegre, RS: Artmed.

Tabachnick, B. G., \& Fidell, L. S. (2001). Using multivariate statistics. New York: Allyn and Bacon.

Tett, R. P., \& Meyer, J. P. (1993). Job satisfaction, organizational commitment, turnover intention, and turnover: Path analyses based on meta-analytic findings. Personnel Psychology, 46(2), 259293. doi: 10.1111/j.1744-6570.1993.tb00874.x

Valentine, S., Godkin, L., Fleischman, G. M., \& Kidwell, R. (2010). Corporate ethical values, group creativity, job satisfaction and turnover intention: The impact of work context on work response. Journal of Business Ethics, 98(3), 353-372. doi: 10.1007/s10551-010-0554-6

Yin-Fah, B. C., Foon, Y. S., Chee-Leong, L., \& Osman, S. (2010). An Exploratory Study on Turnover Intention among Private Sector Employees. International Journal of Business and Management, 5(8), 57-64. doi: http://dx.doi. org/10.5539/ijbm.v5n8p57
Recebido: 22/11/2016

$1^{a}$ revisão: $19 / 06 / 2017$ Aceite final: 19/06/2017

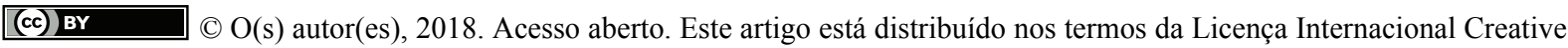
Commons Atribuição 4.0 (http://creativecommons.org/licenses/by/4.0/), que permite o uso, distribuição e reprodução sem restrições em qualquer meio, desde que você dê crédito apropriado ao(s) autor(es) original(ais) e à fonte, fornecer um link para a licença Creative Commons e indicar se as alterações foram feitas. 